\title{
Sentiment cycles in discrete-time homogeneous networks
}

\section{Orlando Gomes*}

Lisbon Accounting and Business School (ISCAL/IPL), Portugal

Business Research Unit (UNIDE/ISCTE-IUL), Portugal

\section{H I G H L I G H T S}

- The paper approaches sentiment transition in a complex network.

- Agents are classified in neutral, optimists and pessimists.

- In continuous-time, the model delivers a stable steady-state outcome.

- In discrete-time, stability holds under a homogeneous network of degree one.

- Endogenous cycles emerge in discrete-time for a connectivity degree larger than one.

\section{A R T I C L E I N F O}

\section{Article history:}

Received 14 June 2014

Received in revised form 1 November 2014

Available online 12 February 2015

\section{Keywords:}

Homogeneous networks

Sentiment-switching

Stability

Endogenous fluctuations

Waves of optimism and pessimism

\begin{abstract}
A B S T R A C T
Consider a network connecting individual agents that are endowed with distinct sentiments or 'views of the world'. Specifically, assume that each node in the network contains an agent that, at a given period $t$, can be found in one of five states: sentiment neutrality, exuberant optimism, non-exuberant optimism, exuberant pessimism and nonexuberant pessimism. Local interaction rules, similar to those one encounters in rumor propagation models, make agents change their sentiment as they contact with others. Under a continuous-time framework, the proposed setting delivers a stable fixed-point equilibrium, meaning that the shares of agents in each sentiment category will converge to constant steady-state levels. The inspection of the same structure of analysis in discretetime indicates that the stability outcome continues to hold when the connectivity degree is equal to 1 . However, this result might change as one considers higher-order connectivity. In this last case, persistent endogenous waves of optimism and pessimism emerge under a reasonable parameterization of the model.
\end{abstract}

(c) 2015 Elsevier B.V. All rights reserved.

\section{Introduction}

Human beings are commonly influenced by the contact they establish with those who occupy adjacent positions in a given social or economic network and, therefore, local interactions constitute a main driver of the micro choices and micro actions that potentially generate observable and meaningful macro patterns. Collective phenomena are typically perceived, as emphasized in Zschaler [1], as the macroscopic outcome emerging from the microscopic and decentralized interaction across a large number of individual units.

In this paper, a complex network is used as the benchmark structure to analyze how the local contact among individuals holding different 'views of the world' determines the overall evolution pattern of the sentiments of a given population.

\footnotetext{
* Correspondence to: Instituto Superior de Contabilidade e Administração de Lisboa (ISCAL/IPL), Av. Miguel Bombarda 20, 1069-035 Lisbon, Portugal.

E-mail address: omgomes@iscal.ipl.pt.
} 
Complex networks are defined by Boccaletti et al. [2] as networks that possess an irregular and evolving structure. They are composed by thousands or millions of nodes that may or may not display different connectivity degrees. ${ }^{1}$ Furthermore, the evolutionary nature of the network implies that agents placed at a given node will potentially change their status as they contact with agents in neighboring nodes.

In the framework under consideration, five categories of individuals will coexist at each time period: those with a neutral sentiment, but who are susceptible of becoming optimistic or pessimistic; non-exuberant optimists and pessimists; and exuberant optimists and pessimists. Local interaction among individuals with different sentiment status will be analyzed through the adaptation of the typical contact rules frequently considered in the analysis of rumor propagation or rumor spreading, as initially designed by Daley and Kendall [3] and Maki and Thompson [4].

Although rumor propagation in social networks is today a prolific line of research, ${ }^{2}$ the adaptation of this theory to the field of sentiment formation and sentiment spreading has been scarce. A recent study, namely Zhao et al. [11], has launched the debate on the subject by proposing a model of sentiment contagion in complex networks, where individual agents face a binary selection mechanism. In their framework, depending on the model's parameterization, only one of two steadystate outcomes is feasible, i.e., in the long-term the entire population has adopted the optimistic view or, alternatively, it has become entirely pessimistic. Differently, in the current paper, the increased detail in terms of sentiment categories and interaction rules will conduct to a more generous set of potential long-term results: the dynamics will contemplate the possibility of a steady-state where part of the population remains optimistic, while others will stay in the pessimistic category; moreover, under certain conditions, the fixed-point steady-state will give place to endogenous fluctuations, that might be interpreted as representing waves of optimism and pessimism at the aggregate level.

The analysis to undertake starts by considering a general complex network in which agents interact locally and potentially change sentiments as the result of the contact they establish with each other. This network is first analyzed in continuoustime. The following results are obtained: (i) a fixed-point steady-state, where positive and constant densities of all sentiment categories persist over time, exists under explicit form and it is determinable; this result holds for a homogeneously mixing population and also for an inhomogeneous structure of interaction; (ii) circumscribing the analysis to the homogeneous network case, it is possible to prove that the steady-state corresponds to a stable equilibrium point.

The results described above are subject to change as one switches from a continuous-time to a discrete-time setting. By evaluating a discrete-time version of the dynamic system, exclusively in the context of homogeneous networks, the following conclusions are reached: (i) stability is confirmed, independently of parameter values, for a network of degree 1 , i.e., for a network where all nodes are connected to a single neighboring node. The array of steady-state values is the same that was calculated in continuous-time; (ii) in networks of a higher connectivity degree, for which each node is connected to $k=2,3, \ldots$ other nodes, the fixed-point result is no longer pervasive; periodic and a-periodic cycles will repeatedly emerge. As a consequence, endogenous fluctuations in the time trajectories of the shares of neutral, optimistic and pessimistic agents are identified as a main result arising in this paper's sentiment-switching model.

The obtained cyclicality result is important because it constitutes a way of justifying how waves of optimism and pessimism eventually arise and persist in any possible economic or social context, as the mere consequence of a simple local interaction process. The only significant premise necessary to achieve this result is that one must avoid taking a homogeneous network of degree 1; a higher than 1 degree of connectivity is all that is required to arrive to the endogenous cycles' outcome. This is a useful result that can be applied, for instance, by economists to introduce an additional source of volatility in their reasoning about short-run macro behavior: animal spirits, in the form of alternating dominant optimism and dominant pessimism, emerge by the contact among neighboring agents that follow simple pre-specified rules of motion.

The remaining part of the paper is organized as follows. Section 2 is dedicated to a brief characterization of the structure of uncorrelated inhomogeneous and homogeneous networks and of the typical dynamics that occur within them. Section 3 introduces sentiment categories, thus giving a concrete substance to the assumed network structure and network relations. In Section 4, the steady-state of the sentiment model under continuous-time is derived and discussed; the respective stability is also evaluated. Section 5 approaches the dynamics under discrete-time and identifies the circumstances in which endogenous fluctuations are formed and sustained over time. Section 6 concludes.

\section{Network dynamics}

Consider a network of social relations, composed by a large number of nodes and by links that establish a connectivity pattern across nodes. The nodes represent individual agents, who can adopt distinct perspectives or interpretations about some socio-economic event. The network is undirected, in the sense that the links do not impose a pre-specified order through which an agent in one node exerts influence over another. The links provide the necessary tool for local interaction to occur; it is this interaction that allows each agent to eventually change her perspective or state, as she suffers the influence of an agent positioned in an adjacent or neighboring node.

\footnotetext{
${ }^{1}$ If every node in the network exhibits an identical connectivity degree, i.e., if there is a same number of links emanating from each node to the other nodes, the network is called homogeneous; otherwise, it acquires the designation of inhomogeneous.

2 Some of the most representative studies published in this area in the last few years include Zanette [5], Thompson et al. [6], Nekovee et al. [7], Huo et al. [8], Zhao et al. [9] and Wang et al. [10].
} 
Assume, for now, that agents across the network may be either at state $x$ or at state $y$. Take a representative node $j$ and consider that, at a given date $t$, this node is in state $x$. Next, consider a local interaction rule according to which the mentioned agent evolves to state $y$, with probability $\lambda$, whenever she establishes contact with an agent in state $y$. Symbolically,

$$
x+y \stackrel{\lambda}{\longrightarrow} y+y .
$$

In Nekovee et al. [7], the framework of interacting Markov chains is employed to determine the average transition probability from one state to the other within a pre-specified time interval $[t, t+\Delta t]$. This average transition probability will differ across connectivity classes if the network is inhomogeneous. In the mentioned study, the average transition probability from state $x$ to state $y$ for nodes with connectivity degree $k$ (i.e., nodes with $k$ links to other nodes) is formally derived and it is expressed in the following way,

$$
p_{k, t}=\left(1-\lambda \Delta t \sum_{k^{\prime}} P\left(k^{\prime} \mid k\right) y_{k^{\prime}, t}\right)^{k} .
$$

In expression (1), $y_{k^{\prime}, t}$ corresponds to the density of nodes, of degree $k^{\prime}$, that are in state $y$. Relatively to $P\left(k^{\prime} \mid k\right)$, this is known as the degree-degree correlation function and it represents the weight of each connectivity class in the contacts established by the agents located at nodes with connectivity degree $k$.

The change in the expected value of the share of nodes in state $x$ belonging to connectivity class $k$ from $t$ to $t+\Delta t$ will then be

$$
x_{k, t+\Delta t}-x_{k, t}=-x_{k, t}\left(1-p_{k, t}\right)
$$

or, equivalently,

$$
x_{k, t+\Delta t}-x_{k, t}=-x_{k, t}\left[1-\left(1-\lambda \Delta t \sum_{k^{\prime}} P\left(k^{\prime} \mid k\right) y_{k^{\prime}, t}\right)^{k}\right] .
$$

The previous expression might be maintained in discrete-time taking consecutive time periods $t=1,2, \ldots$,

$$
x_{k, t+1}-x_{k, t}=-x_{k, t}\left[1-\left(1-\lambda \sum_{k^{\prime}} P\left(k^{\prime} \mid k\right) y_{k^{\prime}, t}\right)^{k}\right]
$$

or transformed into a continuous-time rule of motion by assuming $\Delta t \rightarrow 0$,

$$
\dot{x}(k, t)=-k \lambda x(k, t) \sum_{k^{\prime}} P\left(k^{\prime} \mid k\right) y\left(k^{\prime}, t\right) .
$$

After defining the transition rule from one state to another, one needs to characterize the structure of the underlying network, since it is the type of network that will determine the exact form of function $P\left(k^{\prime} \mid k\right)$, which has a decisive role in the established transition rule. An uncorrelated network is assumed, meaning that the degree-degree correlations take the form

$$
P\left(k^{\prime} \mid k\right)=\frac{k^{\prime} P\left(k^{\prime}\right)}{\langle k\rangle}
$$

with $\langle k\rangle$ the average degree and $P\left(k^{\prime}\right)$ the degree distribution of $k^{\prime}$, i.e., the share of nodes in the graph holding degree $k^{\prime}$. Therefore,

$$
\sum_{k^{\prime}} P\left(k^{\prime} \mid k\right) y_{k^{\prime}, t}=\sum_{k^{\prime}} \frac{k^{\prime} P\left(k^{\prime}\right)}{\langle k\rangle} y_{k^{\prime}, t}=\frac{\sum_{k^{\prime}}\left[k^{\prime} P\left(k^{\prime}\right) y_{k^{\prime}, t}\right]}{\sum_{k^{\prime}} k^{\prime} P\left(k^{\prime}\right)} .
$$

If the network is homogeneous, i.e., if there is only one connectivity class, then $\sum_{k^{\prime}} P\left(k^{\prime} \mid k\right) y_{k^{\prime}, t}=y_{k, t}$. In this specific case, expressions (4) and (5) are reduced to

$$
\begin{aligned}
& x_{k, t+1}-x_{k, t}=-x_{k, t}\left[1-\left(1-\lambda y_{k, t}\right)^{k}\right] \\
& \dot{x}(k, t)=-k \lambda x(k, t) y(k, t) .
\end{aligned}
$$




\section{A sentiment model: local interaction rules}

Agents located at the nodes of the network will belong, under the proposed setting, to one of five different categories, namely

- $x$ : agents with a neutral sentiment, susceptible of becoming either optimistic or pessimistic;

- $y^{\omega}$ : exuberant optimists;

- $y^{\zeta}$ : exuberant pessimists;

- $z^{\omega}$ : non-exuberant optimists;

- $z^{\zeta}$ : non-exuberant pessimists.

The above variables correspond to shares of the entire population, such that $x+y^{\omega}+y^{\zeta}+z^{\omega}+z^{\zeta}=1$. Global shares of optimists and pessimists are, respectively, $\omega \equiv y^{\omega}+z^{\omega}$ and $\zeta \equiv y^{\zeta}+z^{\zeta}$.

Agents in each category might change class through the contact with others. The transition rules to adopt are adapted from rumor propagation theory, where three categories of agents typically coexist: susceptibles, spreaders and stiflers. When a susceptible meets a spreader, this last one transmits, with a given probability, the rumor to the susceptible, who becomes a spreader as well. When spreaders interact with other spreaders or with stiflers they will probably evolve to the stifler state. Eventually, stiflers on a given rumor end up by forgetting the rumor and entering again into the susceptible class, probably for a new or modified rumor; this is likely to happen when a stifler interacts with a susceptible individual.

In the sentiment setting proposed in this paper, the analogy with the rumor spreading framework works as follows. Someone with a neutral sentiment will assume the role of susceptible, in the sense that she can be 'infected' with the optimistic or with the pessimistic sentiment. This occurs, with a given probability, when the neutral individual meets with a true believer of such sentiment, i.e., with someone who has an exuberant attitude about it. The intuition underlying this process is that those with no clearly defined sentiment may fall under the influence of those who are strongly committed in following what they consider the most adequate view of the world. The enthusiastic attitude of the exuberant agents, both optimistic or pessimistic, makes them to act as spreaders; in fact, they will want to spread their belief on a given sentiment to those who, at a given time moment, do not hold any discernible sentiment (the neutrals).

If instead of interacting with a neutral agent, the exuberant individual enters in contact with another exuberant or with a non-exuberant in the same sentiment class, exuberance tends to fade away, with a given probability, giving place to a nonexuberance sentiment. Therefore, non-exuberant optimists and pessimists acquire the role of stiflers: those who share the sentiment, but are no longer sufficiently enthusiastic to spread it. On the contrary, those who were exuberant will typically lose enthusiasm as they interact with other individuals sharing an identical sentiment.

Finally, if someone is in the possession of a given sentiment, but does not support it in an exuberant way, she will probably drop the belief and will fall to a state of neutrality, when meeting another agent for whom there is indifference relatively to any of the sentiments.

Obviously, besides the contact rules described above, one could consider other interactions. For instance, we are ignoring the possibility of an optimist (exuberant or not) and a pessimist (exuberant or not) to eventually influence each other when meeting. The relatively small set of possible relevant interactions one effectively considers allows to keep the analysis at a tractable level, while simultaneously offering a plausible framework to address how individuals change their sentiments over time, as they establish contact with others. The sequence neutrality $\rightarrow$ exuberance $\rightarrow$ non-exuberance $\rightarrow$ neutrality delivers a sensible description of the individuals 'mood swings' and, at the same time, allows for assuming a commuting state, which is neutrality; each time an agent arrives to the neutrality state, one of two steps might follow: she can return to the sentiment category in which she was before or, if meeting an exuberant supporter of the opposite sentiment, she can make a transition to the alternative sentiment class.

Systematizing the logical arguments set forth above, the following rules are adopted:

1. When a neutral agent meets an exuberant optimist or an exuberant pessimist, she will transform herself in an exuberant optimist or in an exuberant pessimist, respectively, with probability $\lambda$, i.e.,

$$
\begin{aligned}
& x+y^{\omega} \stackrel{\lambda}{\longrightarrow} y^{\omega}+y^{\omega} \\
& x+y^{\zeta} \stackrel{\lambda}{\longrightarrow} y^{\zeta}+y^{\zeta} .
\end{aligned}
$$

2. When an exuberant optimist/pessimist enters in contact with another exuberant optimist/pessimist or with a nonexuberant optimist/pessimist, she becomes a non-exuberant optimist/pessimist, with probability $\sigma$,

$$
\begin{aligned}
& y^{\omega}+y^{\omega} \stackrel{\sigma}{\longrightarrow} z^{\omega}+z^{\omega} \\
& y^{\omega}+z^{\omega} \stackrel{\sigma}{\longrightarrow} z^{\omega}+z^{\omega}
\end{aligned}
$$




$$
\begin{aligned}
& y^{\zeta}+y^{\zeta} \stackrel{\sigma}{\longrightarrow} z^{\zeta}+z^{\zeta} \\
& y^{\zeta}+z^{\zeta} \stackrel{\sigma}{\longrightarrow} z^{\zeta}+z^{\zeta} .
\end{aligned}
$$

3. When a non-exuberant optimist/pessimist establishes contact with an individual with a neutral sentiment, the first becomes neutral as well, with probability $\theta$,

$$
\begin{aligned}
& z^{\omega}+x \stackrel{\theta}{\longrightarrow} x+x \\
& z^{\zeta}+x \stackrel{\theta}{\longrightarrow} x+x .
\end{aligned}
$$

Applying to the above rules the transition mechanism described in the previous section, one is able to present a system that provides an overall perspective on the dynamics of changing sentiments. In discrete-time, the system is

$$
\begin{aligned}
& \int x_{k, t+1}-x_{k, t}=-x_{k, t}\left[1-\left(1-\lambda \sum_{k^{\prime}} P\left(k^{\prime} \mid k\right) y_{k^{\prime}, t}^{\omega}\right)^{k}\right]-x_{k, t}\left[1-\left(1-\lambda \sum_{k^{\prime}} P\left(k^{\prime} \mid k\right) y_{k^{\prime}, t}^{\zeta}\right)^{k}\right] \\
& +\left(z_{k, t}^{\omega}+z_{k, t}^{\zeta}\right)\left[1-\left(1-\theta \sum_{k^{\prime}} P\left(k^{\prime} \mid k\right) x_{k^{\prime}, t}\right)^{k}\right] \\
& y_{k, t+1}^{\omega}-y_{k, t}^{\omega}=-y_{k, t}^{\omega}\left[1-\left(1-\sigma\left(\sum_{k^{\prime}} P\left(k^{\prime} \mid k\right) y_{k^{\prime}, t}^{\omega}+\sum_{k^{\prime}} P\left(k^{\prime} \mid k\right) z_{k^{\prime}, t}^{\omega}\right)\right)^{k}\right] \\
& +x_{k, t}\left[1-\left(1-\lambda \sum_{k^{\prime}} P\left(k^{\prime} \mid k\right) y_{k^{\prime}, t}^{\omega}\right)^{k}\right] \\
& y_{k, t+1}^{\zeta}-y_{k, t}^{\zeta}=-y_{k, t}^{\zeta}\left[1-\left(1-\sigma\left(\sum_{k^{\prime}} P\left(k^{\prime} \mid k\right) y_{k^{\prime}, t}^{\zeta}+\sum_{k^{\prime}} P\left(k^{\prime} \mid k\right) z_{k^{\prime}, t}^{\zeta}\right)\right)^{k}\right] \\
& +x_{k, t}\left[1-\left(1-\lambda \sum_{k^{\prime}} P\left(k^{\prime} \mid k\right) y_{k^{\prime}, t}^{\zeta}\right)^{k}\right] \\
& z_{k, t+1}^{\omega}-z_{k, t}^{\omega}=-z_{k, t}^{\omega}\left[1-\left(1-\theta \sum_{k^{\prime}} P\left(k^{\prime} \mid k\right) x_{k^{\prime}, t}\right)^{k}\right] \\
& +y_{k, t}^{\omega}\left[1-\left(1-\sigma\left(\sum_{k^{\prime}} P\left(k^{\prime} \mid k\right) y_{k^{\prime}, t}^{\omega}+\sum_{k^{\prime}} P\left(k^{\prime} \mid k\right) z_{k^{\prime}, t}^{\omega}\right)\right)^{k}\right] \\
& z_{k, t+1}^{\zeta}-z_{k, t}^{\zeta}=-z_{k, t}^{\zeta}\left[1-\left(1-\theta \sum_{k^{\prime}} P\left(k^{\prime} \mid k\right) x_{k^{\prime}, t}\right)^{k}\right] \\
& +y_{k, t}^{\zeta}\left[1-\left(1-\sigma\left(\sum_{k^{\prime}} P\left(k^{\prime} \mid k\right) y_{k^{\prime}, t}^{\zeta}+\sum_{k^{\prime}} P\left(k^{\prime} \mid k\right) z_{k^{\prime}, t}^{\zeta}\right)\right)^{k}\right]
\end{aligned}
$$

with $k=1,2, \ldots, K ; K$ represents the largest number of links that a node in the network possesses. System (10) involves $5 K$ equations and, given its high-dimensionality, the respective dynamics are not straightforward to approach from an analytical point of view.

The determination of the steady-state and the evaluation of the respective stability become feasible once the sentiment dynamics is evaluated in continuous-time. The continuous-time version of system (10) is the following set of differential equations, 


$$
\left\{\begin{array}{c}
\dot{x}(k, t)=-k \lambda x(k, t) \sum_{k^{\prime}} P\left(k^{\prime} \mid k\right) y^{\omega}\left(k^{\prime}, t\right)-k \lambda x(k, t) \sum_{k^{\prime}} P\left(k^{\prime} \mid k\right) y^{\zeta}\left(k^{\prime}, t\right) \\
+k \theta\left[z^{\omega}(k, t)+z^{\zeta}(k, t)\right] \sum_{k^{\prime}} P\left(k^{\prime} \mid k\right) x\left(k^{\prime}, t\right) \\
\dot{y}^{\omega}(k, t)=-k \sigma y^{\omega}(k, t)\left[\sum_{k^{\prime}} P\left(k^{\prime} \mid k\right) y^{\omega}\left(k^{\prime}, t\right)+\sum_{k^{\prime}} P\left(k^{\prime} \mid k\right) z^{\omega}\left(k^{\prime}, t\right)\right] \\
+k \lambda x(k, t) \sum_{k^{\prime}} P\left(k^{\prime} \mid k\right) y^{\omega}\left(k^{\prime}, t\right) \\
\dot{y}^{\zeta}(k, t)=-k \sigma y^{\zeta}(k, t)\left[\sum_{k^{\prime}} P\left(k^{\prime} \mid k\right) y^{\zeta}\left(k^{\prime}, t\right)+\sum_{k^{\prime}} P\left(k^{\prime} \mid k\right) z^{\zeta}\left(k^{\prime}, t\right)\right] \\
+k \lambda x(k, t) \sum_{k^{\prime}} P\left(k^{\prime} \mid k\right) y^{\zeta}\left(k^{\prime}, t\right) \\
\dot{z}^{\omega}(k, t)=-k \theta z^{\omega}(k, t) \sum_{k^{\prime}} P\left(k^{\prime} \mid k\right) x\left(k^{\prime}, t\right) \\
+k \sigma y^{\omega}(k, t)\left[\sum_{k^{\prime}} P\left(k^{\prime} \mid k\right) y^{\omega}\left(k^{\prime}, t\right)+\sum_{k^{\prime}} P\left(k^{\prime} \mid k\right) z^{\omega}\left(k^{\prime}, t\right)\right] \\
\dot{z}^{\zeta}(k, t)=-k \theta z^{\zeta}(k, t) \sum_{k^{\prime}} P\left(k^{\prime} \mid k\right) x\left(k^{\prime}, t\right) \\
+k \sigma y^{\zeta}(k, t)\left[\sum_{k^{\prime}} P\left(k^{\prime} \mid k\right) y^{\zeta}\left(k^{\prime}, t\right)+\sum_{k^{\prime}} P\left(k^{\prime} \mid k\right) z^{\zeta}\left(k^{\prime}, t\right)\right]
\end{array}\right.
$$

Again, $k=1,2, \ldots, K$.

In the next section, the properties of the long-term equilibrium of the system are addressed for the continuous-time case. A steady-state outcome that is independent of the networks' structure is determined. The stability of the equilibrium is discussed and a general stability result is attained under the assumption of link homogeneity.

\section{Equilibrium and stability in continuous-time}

In this section, two propositions are presented. The first relates to the existence and to the determination of the longterm equilibrium. A unique interior steady-state point is computed. The second proposition relates to the stability of the equilibrium. Although it is not feasible to prove stability under the generic form of the continuous-time system (11) via computation of eigenvalues of the Jacobian matrix, such proof becomes possible once a homogeneous network is considered; in this case, the system will be five-dimensional and one will be able to calculate eigenvalues and to determine stability properties through the examination of their signs.

Proposition 1. For $x(k, 0) \neq 0, y^{\omega}(k, 0) \neq 0, y^{\zeta}(k, 0) \neq 0, z^{\omega}(k, 0) \neq 0, z^{\zeta}(k, 0) \neq 0$, the continuous-time version of the sentiment-switching model implies the existence of a unique equilibrium point. The equilibrium point $\left(x^{*},\left(y^{\omega}\right)^{*},\left(y^{\zeta}\right)^{*}\right.$, $\left.\left(z^{\omega}\right)^{*},\left(z^{\zeta}\right)^{*}\right)$ is such that

$$
\begin{aligned}
& x^{*}=\frac{\sigma}{2 \lambda+\sigma} \\
& \left(y^{\omega}\right)^{*}=\left(y^{\zeta}\right)^{*}=\frac{\lambda \theta}{(2 \lambda+\sigma)(\lambda+\theta)} \\
& \left(z^{\omega}\right)^{*}=\left(z^{\zeta}\right)^{*}=\frac{\lambda^{2}}{(2 \lambda+\sigma)(\lambda+\theta)} .
\end{aligned}
$$

Proof. The long-term steady-state is defined, for the continuous-time dynamic system (11) as the point obeying condition $\left(\dot{x}, \dot{y}^{\omega}, \dot{y}^{\zeta}, \dot{z}^{\omega}, \dot{z}^{\zeta}\right)=\overline{0}$, with $\overline{0}$ a five-dimensional vector of zeros. The application of this condition to the set of differential equations in (11) imposes the following equalities,

$$
\begin{aligned}
k \lambda(x(k))^{*} \sum_{k^{\prime}} P\left(k^{\prime} \mid k\right)\left(y^{\omega}\left(k^{\prime}\right)\right)^{*} & =k \sigma\left(y^{\omega}(k)\right)^{*}\left[\sum_{k^{\prime}} P\left(k^{\prime} \mid k\right)\left(y^{\omega}\left(k^{\prime}\right)\right)^{*}+\sum_{k^{\prime}} P\left(k^{\prime} \mid k\right)\left(z^{\omega}\left(k^{\prime}\right)\right)^{*}\right] \\
& =k \theta\left(z^{\omega}(k)\right)^{*} \sum_{k^{\prime}} P\left(k^{\prime} \mid k\right)\left(x\left(k^{\prime}\right)\right)^{*}
\end{aligned}
$$


and

$$
\begin{aligned}
k \lambda(x(k))^{*} \sum_{k^{\prime}} P\left(k^{\prime} \mid k\right)\left(y^{\zeta}\left(k^{\prime}\right)\right)^{*} & =k \sigma\left(y^{\zeta}(k)\right)^{*}\left[\sum_{k^{\prime}} P\left(k^{\prime} \mid k\right)\left(y^{\zeta}\left(k^{\prime}\right)\right)^{*}+\sum_{k^{\prime}} P\left(k^{\prime} \mid k\right)\left(z^{\zeta}\left(k^{\prime}\right)\right)^{*}\right] \\
& =k \theta\left(z^{\zeta}(k)\right)^{*} \sum_{k^{\prime}} P\left(k^{\prime} \mid k\right)\left(x\left(k^{\prime}\right)\right)^{*} .
\end{aligned}
$$

Under the assumption of an uncorrelated network, it follows from (7) that the above sequences of equalities, (12) and (13), are equivalent to

$$
\begin{aligned}
k \lambda(x(k))^{*} \frac{\sum_{k^{\prime}}\left[k^{\prime} P\left(k^{\prime}\right)\left(y^{\omega}\left(k^{\prime}\right)\right)^{*}\right]}{\sum_{k^{\prime}}\left[k^{\prime} P\left(k^{\prime}\right)\right]} & =k \sigma\left(y^{\omega}(k)\right)^{*}\left[\frac{\sum_{k^{\prime}}\left[k^{\prime} P\left(k^{\prime}\right)\left(y^{\omega}\left(k^{\prime}\right)\right)^{*}\right]}{\sum_{k^{\prime}}\left[k^{\prime} P\left(k^{\prime}\right)\right]}+\frac{\sum_{k^{\prime}}\left[k^{\prime} P\left(k^{\prime}\right)\left(z^{\omega}\left(k^{\prime}\right)\right)^{*}\right]}{\sum_{k^{\prime}}\left[k^{\prime} P\left(k^{\prime}\right)\right]}\right] \\
& =k \theta\left(z^{\omega}(k)\right)^{*} \frac{\sum_{k^{\prime}}\left[k^{\prime} P\left(k^{\prime}\right)\left(x\left(k^{\prime}\right)\right)^{*}\right]}{\sum_{k^{\prime}}\left[k^{\prime} P\left(k^{\prime}\right)\right]}
\end{aligned}
$$

and

$$
\begin{aligned}
k \lambda(x(k))^{*} \frac{\sum_{k^{\prime}}\left[k^{\prime} P\left(k^{\prime}\right)\left(y^{\zeta}\left(k^{\prime}\right)\right)^{*}\right]}{\sum_{k^{\prime}}\left[k^{\prime} P\left(k^{\prime}\right)\right]} & =k \sigma\left(y^{\omega}(k)\right)^{*}\left[\frac{\sum_{k^{\prime}}\left[k^{\prime} P\left(k^{\prime}\right)\left(y^{\zeta}\left(k^{\prime}\right)\right)^{*}\right]}{\sum_{k^{\prime}}\left[k^{\prime} P\left(k^{\prime}\right)\right]}+\frac{\sum_{k^{\prime}}\left[k^{\prime} P\left(k^{\prime}\right)\left(z^{\zeta}\left(k^{\prime}\right)\right)^{*}\right]}{\sum_{k^{\prime}}\left[k^{\prime} P\left(k^{\prime}\right)\right]}\right] \\
& =k \theta\left(z^{\zeta}(k)\right)^{*} \frac{\sum_{k^{\prime}}\left[k^{\prime} P\left(k^{\prime}\right)\left(x\left(k^{\prime}\right)\right)^{*}\right]}{\sum_{k^{\prime}}\left[k^{\prime} P\left(k^{\prime}\right)\right]} .
\end{aligned}
$$

Next, assume two different connectivity degrees $\widetilde{k}$ and $\widehat{k}$. The ratio between (14) under $\widetilde{k}$ and (14) under $\widehat{k}$ and the ratio between (15) under $\widetilde{k}$ and (15) under $\widehat{k}$ have correspondence, respectively, in the following equalities,

$$
\frac{\widetilde{k}(x(\tilde{k}))^{*}}{\widehat{k}(x(\widehat{k}))^{*}}=\frac{\widetilde{k}\left(y^{\omega}(\tilde{k})\right)^{*}}{\widehat{k}\left(y^{\omega}(\widehat{k})\right)^{*}}=\frac{\widetilde{k}\left(z^{\omega}(\tilde{k})\right)^{*}}{\widehat{k}\left(z^{\omega}(\widehat{k})\right)^{*}}
$$

and

$$
\frac{\widetilde{k}(x(\widetilde{k}))^{*}}{\widehat{k}(x(\widehat{k}))^{*}}=\frac{\widetilde{k}\left(y^{\zeta}(\widetilde{k})\right)^{*}}{\widehat{k}\left(y^{\zeta}(\widehat{k})\right)^{*}}=\frac{\widetilde{k}\left(z^{\zeta}(\widetilde{k})\right)^{*}}{\widehat{k}\left(z^{\zeta}(\widehat{k})\right)^{*}} .
$$

Condensing in a single expression equalities (16) and (17), it holds that

$$
\frac{(x(\widetilde{k}))^{*}}{(x(\widehat{k}))^{*}}=\frac{\left(y^{\omega}(\tilde{k})\right)^{*}}{\left(y^{\omega}(\widehat{k})\right)^{*}}=\frac{\left(y^{\zeta}(\widetilde{k})\right)^{*}}{\left(y^{\zeta}(\widehat{k})\right)^{*}}=\frac{\left(z^{\omega}(\widetilde{k})\right)^{*}}{\left(z^{\omega}(\widehat{k})\right)^{*}}=\frac{\left(z^{\zeta}(\widetilde{k})\right)^{*}}{\left(z^{\zeta}(\widehat{k})\right)^{*}}, \quad \forall \widetilde{k}, \widehat{k} \in\{1,2, \ldots, K\} .
$$

The sum of the shares of individuals belonging to each sentiment category is equal to 1 for every time period. Obviously, this is also a true statement in the steady-state: $(x(\widetilde{k}))^{*}+\left(y^{\omega}(\widetilde{k})\right)^{*}+\left(y^{\zeta}(\widetilde{k})\right)^{*}+\left(z^{\omega}(\widetilde{k})\right)^{*}+\left(z^{\zeta}(\widetilde{k})\right)^{*}=1$. To proceed, note that one can rearrange this last expression and present it under the form

$$
(x(\widetilde{k}))^{*}\left[1+\frac{\left(y^{\omega}(\tilde{k})\right)^{*}}{(x(\widetilde{k}))^{*}}+\frac{\left(y^{\zeta}(\tilde{k})\right)^{*}}{(x(\widetilde{k}))^{*}}+\frac{\left(z^{\omega}(\widetilde{k})\right)^{*}}{(x(\widetilde{k}))^{*}}+\frac{\left(z^{\zeta}(\tilde{k})\right)^{*}}{(x(\widetilde{k}))^{*}}\right]=1 .
$$

Resorting to the already established equalities, namely the ones in (18), condition (19) is also equivalent to

$$
(x(\tilde{k}))^{*}\left[1+\frac{\left(y^{\omega}(\widehat{k})\right)^{*}}{(x(\widehat{k}))^{*}}+\frac{\left(y^{\zeta}(\widehat{k})\right)^{*}}{(x(\widehat{k}))^{*}}+\frac{\left(z^{\omega}(\widehat{k})\right)^{*}}{(x(\widehat{k}))^{*}}+\frac{\left(z^{\zeta}(\widehat{k})\right)^{*}}{(x(\widehat{k}))^{*}}\right]=1
$$

and, hence, $(x(\widetilde{k}))^{*}\left[1+\frac{1-(x(\widehat{k}))^{*}}{(x(\widehat{k}))^{*}}\right]=1$. This last relation, in turn, implies the existence of a steady-state value $(x)^{*} \equiv$ $(x(\widetilde{k}))^{*}=(x(\widehat{k}))^{*}$. Consequently, all the other ratios between the same sentiment shares across connectivity classes must be, 
as well, equal to 1 , given (18), and therefore it is admissible to define the following equilibrium shares: $\left(y^{\omega}\right)^{*} \equiv\left(y^{\omega}(\widetilde{k})\right)^{*}=$ $\left(y^{\omega}(\widehat{k})\right)^{*} ;\left(y^{\zeta}\right)^{*} \equiv\left(y^{\zeta}(\widetilde{k})\right)^{*}=\left(y^{\zeta}(\widehat{k})\right)^{*} ;\left(z^{\omega}\right)^{*} \equiv\left(z^{\omega}(\widetilde{k})\right)^{*}=\left(z^{\omega}(\widehat{k})\right)^{*} ;\left(z^{\zeta}\right)^{*} \equiv\left(z^{\zeta}(\widetilde{k})\right)^{*}=\left(z^{\zeta}(\widehat{k})\right)^{*}$.

The previous result indicates that the shares of agents in each sentiment category are, in the steady-state, identical independently of the connectivity degree. This is a fundamental result because it determines that, in equilibrium,

$$
\begin{aligned}
& \sum_{k^{\prime}} P\left(k^{\prime} \mid k\right)\left(x\left(k^{\prime}\right)\right)^{*}=x^{*} \sum_{k^{\prime}} P\left(k^{\prime} \mid k\right)=x^{*} \\
& \sum_{k^{\prime}} P\left(k^{\prime} \mid k\right)\left(y^{\omega}\left(k^{\prime}\right)\right)^{*}=\left(y^{\omega}\right)^{*} \sum_{k^{\prime}} P\left(k^{\prime} \mid k\right)=\left(y^{\omega}\right)^{*} \\
& \sum_{k^{\prime}} P\left(k^{\prime} \mid k\right)\left(y^{\zeta}\left(k^{\prime}\right)\right)^{*}=\left(y^{\zeta}\right)^{*} \sum_{k^{\prime}} P\left(k^{\prime} \mid k\right)=\left(y^{\zeta}\right)^{*} \\
& \sum_{k^{\prime}} P\left(k^{\prime} \mid k\right)\left(z^{\omega}\left(k^{\prime}\right)\right)^{*}=\left(z^{\omega}\right)^{*} \sum_{k^{\prime}} P\left(k^{\prime} \mid k\right)=\left(z^{\omega}\right)^{*} \\
& \sum_{k^{\prime}} P\left(k^{\prime} \mid k\right)\left(z^{\zeta}\left(k^{\prime}\right)\right)^{*}=\left(z^{\zeta}\right)^{*} \sum_{k^{\prime}} P\left(k^{\prime} \mid k\right)=\left(z^{\zeta}\right)^{*} .
\end{aligned}
$$

The above relations and results imply that the continuous-time version of the sentiment-switching model can be reduced, in terms of steady-state evaluation, to the system composed by the following collection of equations:

$$
\left\{\begin{array}{l}
\lambda x^{*}\left(\left(y^{\omega}\right)^{*}+\left(y^{\zeta}\right)^{*}\right)=\theta x^{*}\left(\left(z^{\omega}\right)^{*}+\left(z^{\zeta}\right)^{*}\right) \\
\sigma\left(y^{\omega}\right)^{*}\left(\left(y^{\omega}\right)^{*}+\left(z^{\omega}\right)^{*}\right)=\lambda x^{*}\left(y^{\omega}\right)^{*} \\
\sigma\left(y^{\zeta}\right)^{*}\left(\left(y^{\zeta}\right)^{*}+\left(z^{\zeta}\right)^{*}\right)=\lambda x^{*}\left(y^{\zeta}\right)^{*} \\
\theta x^{*}\left(z^{\omega}\right)^{*}=\sigma\left(y^{\omega}\right)^{*}\left(\left(y^{\omega}\right)^{*}+\left(z^{\omega}\right)^{*}\right) \\
\theta x^{*}\left(z^{\zeta}\right)^{*}=\sigma\left(y^{\zeta}\right)^{*}\left(\left(y^{\zeta}\right)^{*}+\left(z^{\zeta}\right)^{*}\right) .
\end{array}\right.
$$

From the above system, excluding the possibility of a long-run scenario in which the sentiment shares are equal to zero, what is guaranteed for the assumed initial conditions, it holds true that $\left(y^{\omega}\right)^{*}+\left(z^{\omega}\right)^{*}=\left(y^{\zeta}\right)^{*}+\left(z^{\zeta}\right)^{*}$, i.e., in the steady-state the shares of optimists and pessimists are identical. Therefore, the expression concerning the sum of all the steady-state sentiment shares can be displayed as $x^{*}+2\left(\left(y^{\omega}\right)^{*}+\left(z^{\omega}\right)^{*}\right)=x^{*}+2\left(\left(y^{\zeta}\right)^{*}+\left(z^{\zeta}\right)^{*}\right)=1$ or, equivalently, $\left(y^{\omega}\right)^{*}+\left(z^{\omega}\right)^{*}=\left(y^{\zeta}\right)^{*}+\left(z^{\zeta}\right)^{*}=\frac{1-x^{*}}{2}$. Taking into account the second or third equations of system (21), the following relation emerges: $\sigma \frac{1-x^{*}}{2}=\lambda x^{*}$. Solving with respect to the neutrality share equilibrium value, one gets

$$
x^{*}=\frac{\sigma}{2 \lambda+\sigma} .
$$

To arrive to the other variables' steady-state values, first replace $x^{*}$ into the sums of optimists and pessimists, to observe that

$$
\left(y^{\omega}\right)^{*}+\left(z^{\omega}\right)^{*}=\left(y^{\zeta}\right)^{*}+\left(z^{\zeta}\right)^{*}=\frac{\lambda}{2 \lambda+\sigma} .
$$

Next, resorting to the last two equations of the steady-state system (21), the following equalities emerge,

$$
\begin{aligned}
& \theta\left(z^{\omega}\right)^{*}=\lambda\left(\frac{\lambda}{2 \lambda+\sigma}-\left(z^{\omega}\right)^{*}\right) \\
& \theta\left(z^{\zeta}\right)^{*}=\lambda\left(\frac{\lambda}{2 \lambda+\sigma}-\left(z^{\zeta}\right)^{*}\right) .
\end{aligned}
$$

Solving each of the above equations, (24) and (25), with respect to the shares of non-exuberant individuals, the steady-state outcome for the non-exuberant shares is obtained,

$$
\left(z^{\omega}\right)^{*}=\left(z^{\zeta}\right)^{*}=\frac{\lambda^{2}}{(2 \lambda+\sigma)(\lambda+\theta)} .
$$

Finally, one may recover the steady-state sums of overall shares of optimists and pessimists to encounter the steady-state levels of the shares of exuberant optimists and exuberant pessimists,

$$
\left(y^{\omega}\right)^{*}=\left(y^{\zeta}\right)^{*}=\frac{\lambda}{2 \lambda+\sigma}-\left(z^{\omega}\right)^{*}=\frac{\lambda}{2 \lambda+\sigma}-\left(z^{\zeta}\right)^{*}=\frac{\lambda \theta}{(2 \lambda+\sigma)(\lambda+\theta)} .
$$


Results (22), (26) and (27) furnish the long-term equilibrium shares of each sentiment category, as presented in the proposition.

Steady-state stability is approachable through the linearization of system (11) in the vicinity of the derived equilibrium point. Under its general form, this is a system of dimension $5 K$, that is not feasible to analyze, in what concerns the determination of stability conditions, given its high dimensionality. To allow for the tractability of the model, one now considers a homogeneous network of a given $k$ degree.

It is the sign of each of the eigenvalues of the Jacobian matrix of the system that will indicate what kind of stability result characterizes the set of dynamic relations under consideration. In continuous-time, full stability implies that the referred eigenvalues are negative, while instability holds for positive eigenvalues. If part of the eigenvalues are negative and the other part has a positive sign, the system is saddle-path stable with the dimensionality of the stable trajectory given by the number of negative eigenvalues.

In the current case, given the homogeneity assumption, system (11) is five-dimensional; therefore, five eigenvalues can be extracted from the mentioned Jacobian matrix. Nevertheless, one should keep in mind an important detail: in reality, the system under consideration is, in fact, of dimension 4 , since $x+y^{\omega}+y^{\zeta}+z^{\omega}+z^{\zeta}=1$. This constraint implies that one of the differential equations is redundant and that the system may be analyzed in a four-dimensional version, once we replace one of the variables by 1 minus the sum of the others. Keeping the five equations will imply, in practical terms, that one of the eigenvalues to be determined will reflect the existence of a bifurcation dimension, i.e., one of the eigenvalues must necessarily be equal to zero. Hence, to arrive to a stability result according to which the sentiment shares converge to their steady-state levels, the following outcome must be accomplished: taking a $5 \times 5$ Jacobian matrix, one of the corresponding eigenvalues will be equal to zero and the other four must be negative real values or complex roots with a negative real part.

Proposition 2. Consider the sentiment model in its continuous-time homogeneous network version. The steady-state point $\left(x^{*},\left(y^{\omega}\right)^{*},\left(y^{\zeta}\right)^{*},\left(z^{\omega}\right)^{*},\left(z^{\zeta}\right)^{*}\right)$ is locally stable.

Proof. To approach the stability of the equilibrium, one must linearize system (11) in the vicinity of the steady-state. In matricial form, the linearized system is

$$
\left[\begin{array}{c}
\dot{x}(k, t) \\
\dot{y}^{\omega}(k, t) \\
\dot{y}^{\zeta}(k, t) \\
\dot{z}^{\omega}(k, t) \\
\dot{z}^{\zeta}(k, t)
\end{array}\right]=J \cdot\left[\begin{array}{c}
x(k, t)-x^{*} \\
y^{\omega}(k, t)-\left(y^{\omega}\right)^{*} \\
y^{\zeta}(k, t)-\left(y^{\zeta}\right)^{*} \\
z^{\omega}(k, t)-\left(z^{\omega}\right)^{*} \\
z^{\zeta}(k, t)-\left(z^{\zeta}\right)^{*}
\end{array}\right]
$$

with $J$ the Jacobian matrix of the system. The calculus of the derivatives of each equation with respect to each endogenous variable, and the evaluation of the obtained results in the steady-state point, yield

$$
J=\frac{k}{(2 \lambda+\sigma)(\lambda+\theta)}\left[\begin{array}{ccccc}
0 & -\lambda \sigma(\lambda+\theta) & -\lambda \sigma(\lambda+\theta) & \sigma \theta(\lambda+\theta) & \sigma \theta(\lambda+\theta) \\
\lambda^{2} \theta & -\lambda \sigma \theta & 0 & -\lambda \sigma \theta & 0 \\
\lambda^{2} \theta & 0 & -\lambda \sigma \theta & 0 & -\lambda \sigma \theta \\
-\lambda^{2} \theta & \lambda \sigma(\lambda+2 \theta) & 0 & -\sigma \theta^{2} & 0 \\
-\lambda^{2} \theta & 0 & \lambda \sigma(\lambda+2 \theta) & 0 & -\sigma \theta^{2}
\end{array}\right]
$$

Under the specific features of the proposed sentiment model, stability is guaranteed, as mentioned above, if four of the five eigenvalues of $J$ are negative and the other one is equal to zero. Straightforward computation allows to calculate the following eigenvalues:

$$
\begin{aligned}
& \varepsilon_{1}=0 \\
& \varepsilon_{2,3}=-\frac{1}{2} \frac{\sigma k}{2 \lambda+\sigma}\left(\theta \pm \sqrt{\theta^{2}-4 \lambda \theta}\right) \\
& \varepsilon_{4,5}=-\frac{1}{2} \frac{\sigma k}{2 \lambda+\sigma}\left(\theta \pm \sqrt{\theta^{2}-4 \lambda \theta\left(\frac{2 \lambda+\sigma}{\sigma}\right)}\right) .
\end{aligned}
$$

Expressions $\varepsilon_{2}$ to $\varepsilon_{5}$ correspond either to complex conjugate eigenvalues or to real eigenvalues. If $\theta<4 \lambda$ or $\theta<$ $4 \lambda\left(\frac{2 \lambda+\sigma}{\sigma}\right)$, the eigenvalues are complex roots for which it is straightforward to observe the existence of negative real parts. In the opposite circumstance, in which eigenvalues are real roots, it is also clear that they must be negative quantities, since $\theta>\sqrt{\theta^{2}-4 \lambda \theta}$ and $\theta>\sqrt{\theta^{2}-4 \lambda \theta\left(\frac{2 \lambda+\sigma}{\sigma}\right)}$, under the constraint on parameters $\lambda, \sigma, \theta \in(0,1)$.

Therefore, $\varepsilon_{2,3,4,5}<0$, meaning that local stability holds, i.e., that convergence from any initial state in the steady-state vicinity towards the steady-state point will necessarily take place. 


\section{The homogeneous network in discrete time: the emergence of fluctuations}

The inspection of the sentiment-switching model in continuous-time has shown the existence of a unique steady-state, when it is excluded the possibility of shares of individuals that at $t=0$ are equal to zero. As proven, this interior equilibrium is stable under a homogeneous network setting. The analysis in discrete-time, to be undertaken in this section, reveals that the stability result might fail, even in the context of homogeneous networks. Specifically, if one assumes $k \neq 1$ the stability result is no longer universal.

The adoption of a continuous or of a discrete notion of time depends on the subject under examination and on the goals of the analysis. In what concerns the specific case of sentiment networks, the precedent section has clearly demonstrated that a continuous-time approach is useful from the point of view of analytical tractability, allowing to reach relevant dynamic results that are robust to changes on parameter values. However, most of the social and economic phenomena relating sentiment evolution involves discrete decisions and discrete processes in time. Direct contact among human agents occurs intermittently and this alternative notion of time is apparently more adequate to deal with the kind of issue approached in this study, namely if one wants to confront the model's predictions with empirical evidence: empirical data is necessarily discrete.

Furthermore, it is the discrete-time version of the model that will unveil the potential existence of endogenous fluctuations, meaning that sentiment cycles typically emerge when assuming a sequence of time periods instead of a continuous time flow. In the specific case of the model under examination, a discrete notion of time is central to justify a process of purely endogenous sentiment cycles, where changes on sentiments are not triggered by any external force, but solely by the mechanism of interaction one has conceived.

The system in discrete-time is analytically tractable only for $k=1$; for higher-order connectivity, results on the steadystate and its stability can be found only through numerical simulation. Let us begin by approaching the connectivity degree 1 case. $^{3}$

Under $k=1$, the relevant dynamic system is a simplified version of (10),

$$
\left\{\begin{array}{l}
x_{t+1}-x_{t}=-\lambda x_{t}\left(y_{t}^{\omega}+y_{t}^{\zeta}\right)+\theta\left(z_{t}^{\omega}+z_{t}^{\zeta}\right) x_{t} \\
y_{t+1}^{\omega}-y_{t}^{\omega}=-\sigma y_{t}^{\omega}\left(y_{t}^{\omega}+z_{t}^{\omega}\right)+\lambda x_{t} y_{t}^{\omega} \\
y_{t+1}^{\zeta}-y_{t}^{\zeta}=-\sigma y_{t}^{\zeta}\left(y_{t}^{\zeta}+z_{t}^{\zeta}\right)+\lambda x_{t} y_{t}^{\zeta} \\
z_{t+1}^{\omega}-z_{t}^{\omega}=-\theta z_{t}^{\omega} x_{t}+\sigma y_{t}^{\omega}\left(y_{t}^{\omega}+z_{t}^{\omega}\right) \\
z_{t+1}^{\zeta}-z_{t}^{\zeta}=-\theta z_{t}^{\zeta} x_{t}+\sigma y_{t}^{\zeta}\left(y_{t}^{\zeta}+z_{t}^{\zeta}\right) .
\end{array}\right.
$$

Proposition 3. The sentiment model in discrete-time, under $k=1$, possesses a unique steady-state point for $x_{0} \neq 0, y_{0}^{\omega} \neq$ $0, y_{0}^{\zeta} \neq 0, z_{0}^{\omega} \neq 0, z_{0}^{\zeta} \neq 0$. This steady-state is identical to the one derived in continuous-time.

Proof. Let $x^{*} \equiv x_{t+1}=x_{t} ;\left(y^{\omega}\right)^{*} \equiv y_{t+1}^{\omega}=y_{t}^{\omega} ;\left(y^{\zeta}\right)^{*} \equiv y_{t+1}^{\zeta}=y_{t}^{\zeta} ;\left(z^{\omega}\right)^{*} \equiv z_{t+1}^{\omega}=z_{t}^{\omega} ;\left(z^{\zeta}\right)^{*} \equiv z_{t+1}^{\zeta}=z_{t}^{\zeta}$ be the steady-state values one can obtain from (29). From the second and third equations of system (29) it holds true that $\left(y^{\omega}\right)^{*}+\left(z^{\omega}\right)^{*}=\left(y^{\zeta}\right)^{*}+\left(z^{\zeta}\right)^{*}=\frac{\lambda}{\sigma} x^{*}$. The condition representing the sum of the various sentiment shares is, then, presentable as $x^{*}+2\left(\left(y^{\omega}\right)^{*}+\left(z^{\omega}\right)^{*}\right)=x^{*}+2\left(\left(y^{\zeta}\right)^{*}+\left(z^{\zeta}\right)^{*}\right)=1$ or $x^{*}+\frac{\lambda}{\sigma} x^{*}=1$. Consequently, the steady-state value of the neutrality share is, as found for the continuous-time case, $x^{*}=\frac{\sigma}{2 \lambda+\sigma}$.

Next, one proceeds as in the proof of the continuous-time case, first by noting that

$$
\left(y^{\omega}\right)^{*}+\left(z^{\omega}\right)^{*}=\left(y^{\zeta}\right)^{*}+\left(z^{\zeta}\right)^{*}=\frac{\lambda}{2 \lambda+\sigma}
$$

and then by using the last two equations of the system to write the equalities

$$
\begin{aligned}
& \theta\left(z^{\omega}\right)^{*}=\lambda\left(\frac{\lambda}{2 \lambda+\sigma}-\left(z^{\omega}\right)^{*}\right) \\
& \theta\left(z^{\zeta}\right)^{*}=\lambda\left(\frac{\lambda}{2 \lambda+\sigma}-\left(z^{\zeta}\right)^{*}\right) .
\end{aligned}
$$

\footnotetext{
3 Most real-world natural, social or economic phenomena are approachable under the structure of complex networks, namely scale-free networks, where a large number of nodes are poorly connected and a few nodes possess a high degree of connectivity (see, concerning scale-free networks and their practical applicability, Barabási and Albert, [12], and Barabási, [13]). Thus, the case of simple homogeneous networks with $k=1$ is far from being a reasonable description of a world where most networks involve a degree distribution that is power-law shaped. Nevertheless, starting by examining the degree 1 case has the advantage of building a benchmark relatively to which more complex networks can be compared to. Therefore, this simplest case is introduced mainly to serve as an initial reference from which the analysis should start from before proceeding to more sophisticated settings. This is the strategy followed, as well, in the rumor propagation theory, where the analysis of concrete phenomena is also often preceded by the evaluation of degree 1 homogeneous networks (see references in footnote 2 ). As we will observe, complex long-term outcomes only arise precisely when departing from $k=1$ and, thus, when considering a more inter-connected network, what reinforces the argument that $k>1$ cases are those that are truly meaningful in the context of our model.
} 
Table 1

Steady-state values for $\lambda=0.25, \sigma=0.4$

$\theta=0.1$ and different connectivity degrees.

\begin{tabular}{llll}
\hline$k$ & $x^{*}$ & $\left(y^{\omega}\right)^{*},\left(y^{\zeta}\right)^{*}$ & $\left(z^{\omega}\right)^{*},\left(z^{\zeta}\right)^{*}$ \\
\hline 1 & 0.4444 & 0.0794 & 0.1984 \\
2 & 0.4325 & 0.0804 & 0.2033 \\
3 & 0.4205 & 0.0815 & 0.2083 \\
4 & 0.4083 & 0.0827 & 0.2131 \\
5 & 0.3960 & 0.0840 & 0.2180 \\
6 & 0.3838 & 0.0854 & 0.2227 \\
\hline
\end{tabular}

From the above equalities it is straightforward the presentation of the steady-state values of the shares of non-exuberant individuals,

$$
\left(z^{\omega}\right)^{*}=\left(z^{\zeta}\right)^{*}=\frac{\lambda^{2}}{(2 \lambda+\sigma)(\lambda+\theta)} .
$$

Proceeding as before, one also determines the equilibrium values of shares $y_{t}^{\omega}$ and $y_{t}^{\zeta}$,

$$
\left(y^{\omega}\right)^{*}=\left(y^{\zeta}\right)^{*}=\frac{\lambda \theta}{(2 \lambda+\sigma)(\lambda+\theta)} .
$$

The steady-state point will be stable, under the same reasoning one as established in continuous-time, if the respective 5-dimensional linearized system involves one bifurcation dimension and a 4-dimensional stable space. In discrete-time this signifies that one of the eigenvalues of the Jacobian matrix must be equal to -1 or equal to 1 , while the other four lie inside the unit circle. This is precisely the result one finds through the evaluation of the linearized system.

Proposition 4. In the discrete-time sentiment model under the homogeneous network scenario with $k=1$, the steady-state point is locally stable.

Proof. The linearization of system (29) in the vicinity of the steady-state conducts to the matricial representation,

$$
\left[\begin{array}{c}
x_{t+1}-x^{*} \\
y_{t+1}^{\omega}-\left(y^{\omega}\right)^{*} \\
y_{t+1}^{\zeta}-\left(y^{\zeta}\right)^{*} \\
z_{t+1}^{\omega}-\left(z^{\omega}\right)^{*} \\
z_{t+1}^{\zeta}-\left(z^{\zeta}\right)^{*}
\end{array}\right]=J \cdot\left[\begin{array}{c}
x_{t}-x^{*} \\
y_{t}^{\omega}-\left(y^{\omega}\right)^{*} \\
y_{t}^{\zeta}-\left(y^{\zeta}\right)^{*} \\
z_{t}^{\omega}-\left(z^{\omega}\right)^{*} \\
z_{t}^{\zeta}-\left(z^{\zeta}\right)^{*}
\end{array}\right]
$$

Again, $J$ corresponds to a $5 \times 5$ matrix containing the derivatives of each equation relatively to each endogenous variable, duly evaluated in the steady-state. In this specific case,

$$
\begin{gathered}
J=\frac{1}{(2 \lambda+\sigma)(\lambda+\theta)}\left[\begin{array}{ccc}
(2 \lambda+\sigma)(\lambda+\theta) & -\lambda \sigma(\lambda+\theta) & -\lambda \sigma(\lambda+\theta) \\
\lambda^{2} \theta & (2 \lambda+\sigma)(\lambda+\theta)-\lambda \sigma \theta & 0 \\
\lambda^{2} \theta & 0 & (2 \lambda+\sigma)(\lambda+\theta)-\lambda \sigma \theta \\
-\lambda^{2} \theta & \lambda \sigma(\lambda+2 \theta) & 0 \\
-\lambda^{2} \theta & 0 & \lambda \sigma(\lambda+2 \theta) \\
\sigma \theta(\lambda+\theta) & & \sigma \theta(\lambda+\theta) \\
-\lambda \sigma \theta & 0 \\
0 & -\lambda \sigma \theta \\
(2 \lambda+\sigma)(\lambda+\theta)-\sigma \theta^{2} & 0 \\
0 & (2 \lambda+\sigma)(\lambda+\theta)-\sigma \theta^{2}
\end{array}\right] .
\end{gathered}
$$

The eigenvalues of $J$ are straightforward to obtain. They are,

$$
\begin{aligned}
& \varepsilon_{1}=1 ; \quad \varepsilon_{2,3}=1-\frac{1}{2} \frac{\sigma}{2 \lambda+\sigma}\left(\theta \pm \sqrt{\theta^{2}-4 \lambda \theta}\right) \\
& \varepsilon_{4,5}=1-\frac{1}{2} \frac{\sigma}{2 \lambda+\sigma}\left(\theta \pm \sqrt{\theta^{2}-4 \lambda \theta\left(\frac{2 \lambda+\sigma}{\sigma}\right)}\right) .
\end{aligned}
$$

Given admissible parameter values, i.e., $\lambda, \sigma, \theta \in(0,1)$, the examination of the expressions of the eigenvalues indicates that $\varepsilon_{2,3,4,5}$ may be real or complex roots; in any case, constraint $-1<\varepsilon_{2,3,4,5}<1$ is satisfied. Therefore, by finding that 


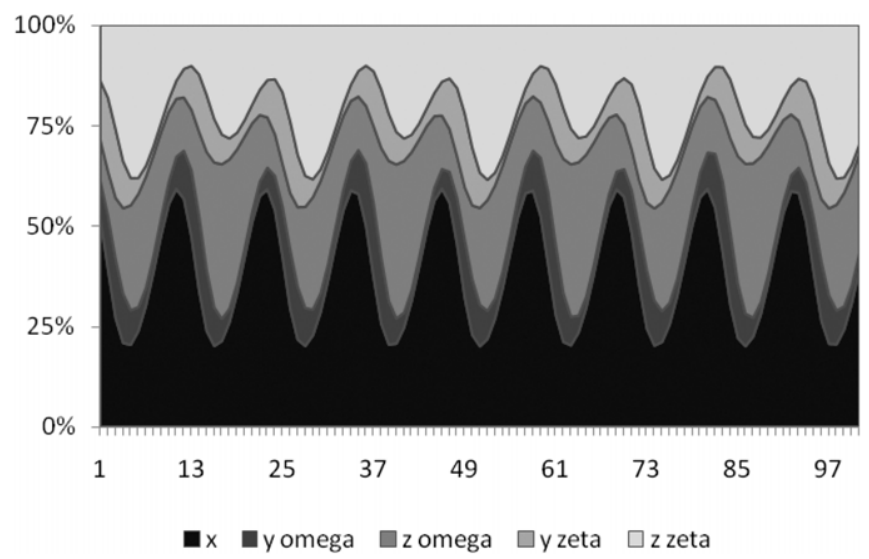

Panel $A: k=7$

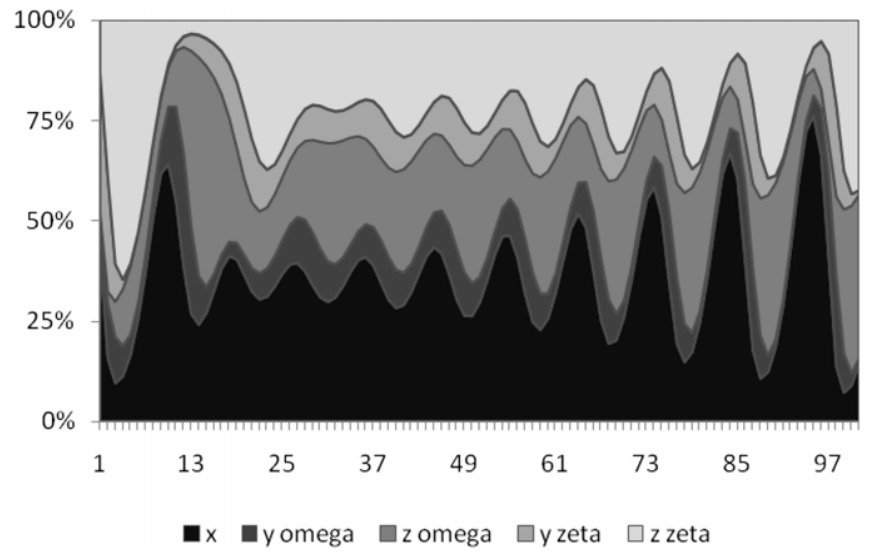

Panel $B: k=9$

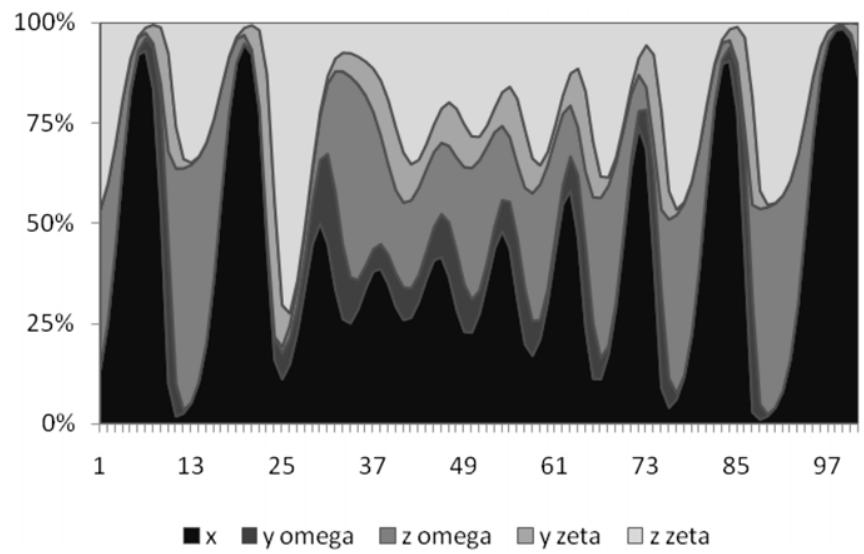

Panel $C: k=11$

Fig. 1. Evolution in time of the density shares $(\lambda=0.25 ; \sigma=0.4 ; \theta=0.1)$. (Note: The five areas in the diagram respect, for the assumed time span displayed in the horizontal axis, to the cumulative percentages of individuals belonging to each of the sentiment categories.)

four eigenvalues lie inside the unit circle while the remainder is equal to 1 , one concludes that the convergence from the initial point towards the steady-state will succeed, i.e., that the dynamic system is stable.

The previous results, namely the set of steady-state values and the stability outcome, change, in discrete-time, once we depart from the $k=1$ case. Although it is not possible to encounter the expressions of the steady-state shares for values of $k$ different from 1 , one can rely on a numerical example to illustrate the differences. Consider the benchmark example $\lambda=0.25, \sigma=0.4, \theta=0.1$, and take any initial values of sentiment densities different from zero. 

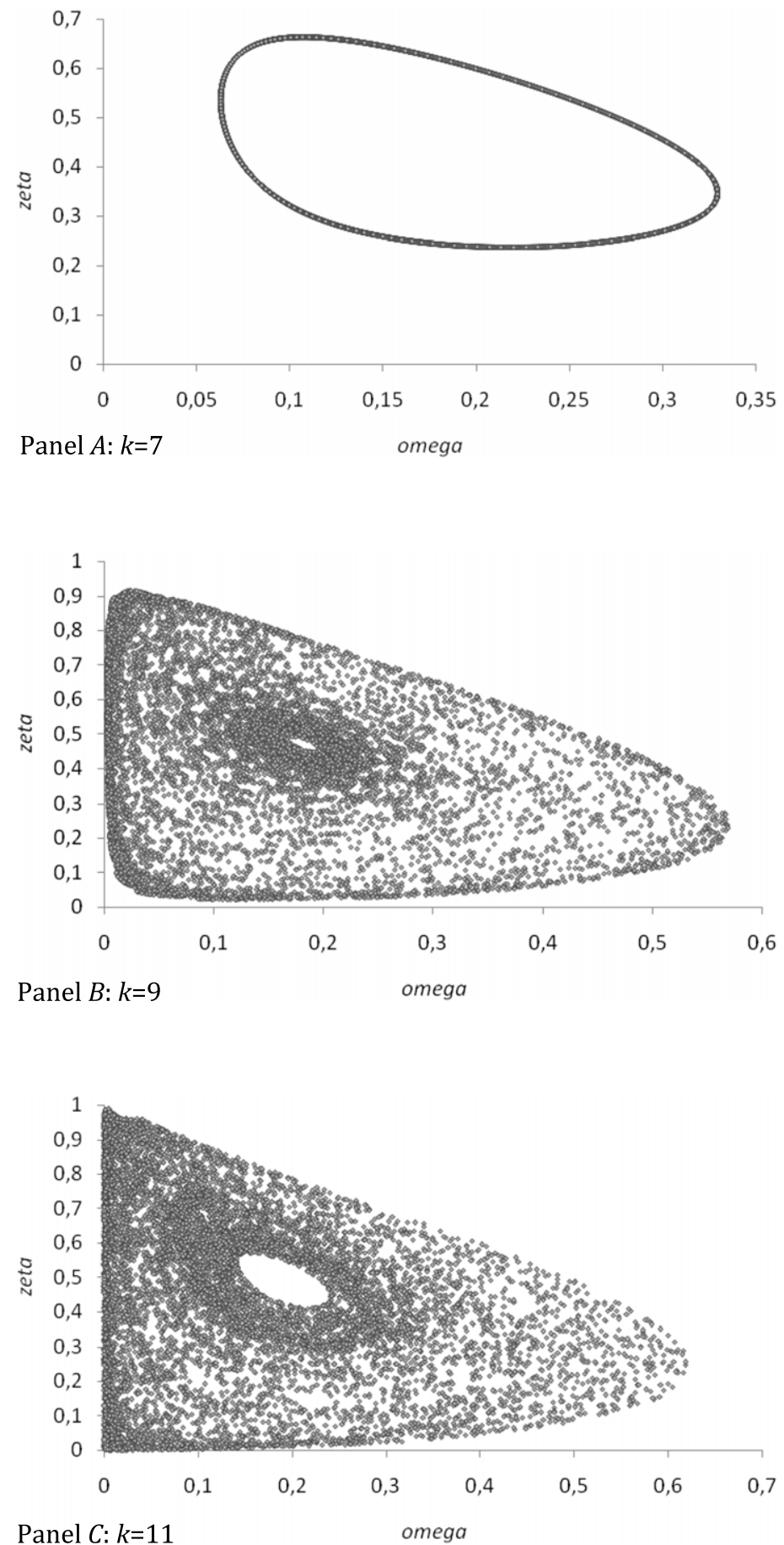

Fig. 2. Long-term attractor between densities of optimists and pessimists $(\lambda=0.25 ; \sigma=0.4 ; \theta=0.1)$.

Table 1 presents the steady-state outcome for the various sentiment shares, for values of $k$ from 1 to 6 . As one observes, these values are not identical across different connectivity degrees. In the case, the share of individuals in the neutrality state falls as $k$ rises, while all the other steady-state sentiment densities will increase. This is an intuitively correct result: a more inter-connected network will imply a faster change from one sentiment class to the other, forcing individuals to remain fewer time in a neutrality position.

The most important result is that stability will no longer be a universal outcome. The connectivity degree and the values of parameters will determine the type of long-term locus. In the benchmark case one has considered, stability will hold for the values of $k$ displayed in the table, but irregular cycles that configure a result of perpetual bounded instability will emerge for 


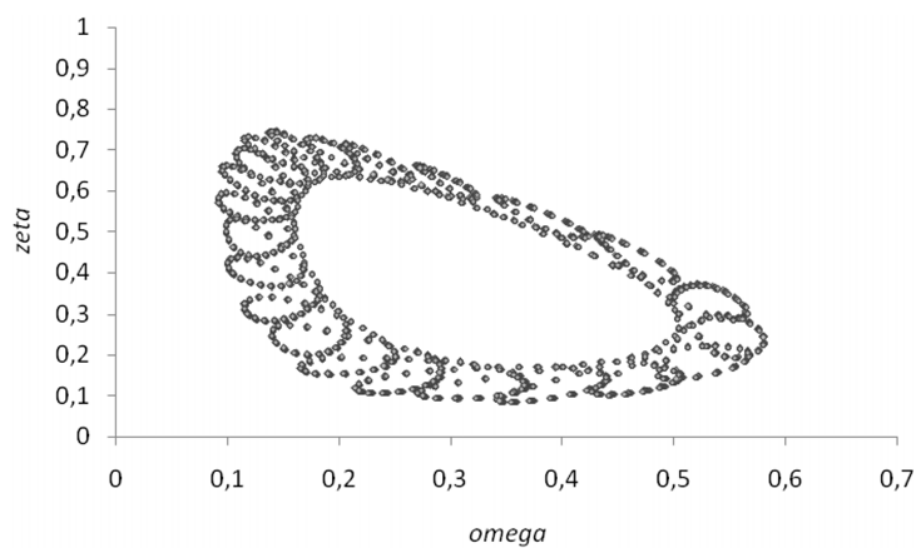

Panel $A$ : $\lambda=0.8 ; \sigma=0.7 ; \theta=0.7 ; k=3$

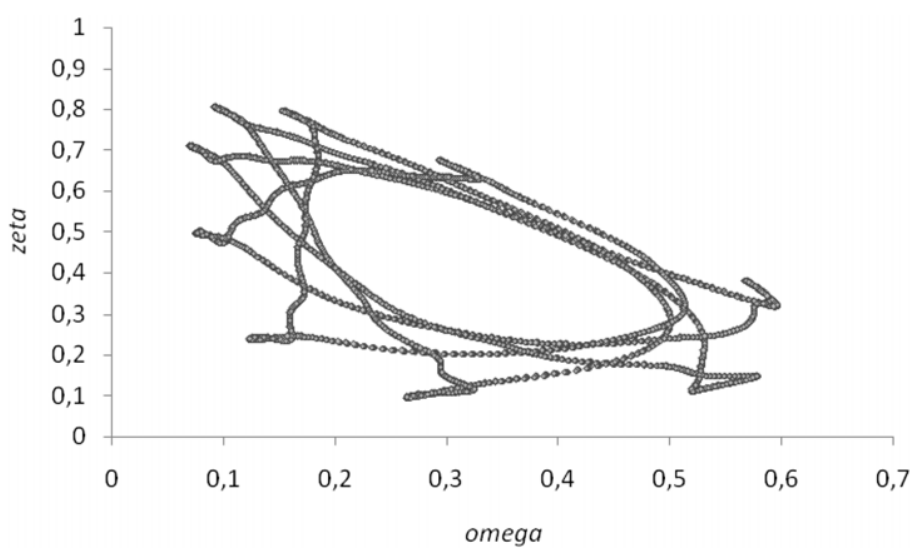

Panel $B: \lambda=0.6 ; \sigma=0.4 ; \theta=0.45 ; k=4$

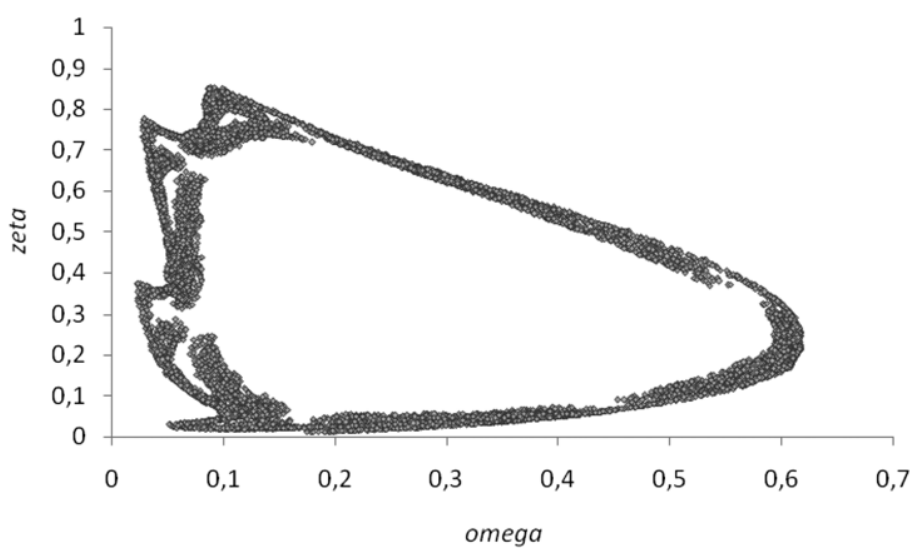

Panel $C: \lambda=0.6 ; \sigma=0.85 ; \theta=0.75 ; k=5$

Fig. 3. Long-term attractor between densities of optimists and pessimists.

higher values of $k$. Figs. 1 and 2 show the time evolution of the density shares and the long-term attractors between densities of optimists and pessimists for $k=7, k=9$ and $k=11$. The presence of endogenous fluctuations is observed. Both the evolution of the shares and the attractors are drawn for long-term observations, after excluding the transient phase. 
Note that in each of the panels of Fig. 1, five areas are represented. These correspond, for a sequence of 100 time periods, to the percentages of the shares of the five sentiment categories (they are displayed cumulatively ones above the others). The figure shows, in all cases, that the percentages of neutral individuals, exuberant and non-exuberant optimists and pessimists fluctuate irregularly over time without any tendency for converging to constant steady-state levels.

Fig. 3 reinforces the illustration of the pervasiveness of the obtained cyclical outcome, by presenting long-term attractors, between shares of optimists and pessimists, for other possible combinations of parameters. Results suggest that the local interaction in a large scale network across agents with different 'views of the world' is likely to culminate in an outcome of deterministic unpredictability.

\section{Conclusion}

The analysis has revealed that, in continuous-time, a sentiment model built upon a complex network delivers a stable steady-state outcome in which constant positive shares of sentiment states coexist in the long-run. This result continues to hold in a discrete-time version of the model for a homogeneous network of connectivity degree 1. However, when assuming higher degrees of connectivity and, thus, a more realistic network, endogenous fluctuations emerge for admissible and reasonable parameter values. Therefore, by conceiving a relatively simple local interaction structure, where individuals holding distinct beliefs or distinct 'views of the world' establish contact and influence each other, one was able to justify the formation of waves of optimism and pessimism. These waves emerge as the direct result of the mechanics of the model, without the need for assuming any external shock.

The findings in this paper are particularly important for macroeconomic analysis, since they may be used to argue in favor of a business cycles interpretation in which animal spirits, in the form of changing sentiments, eventually play a meaningful role. Economic agents often lack the ability to understand the economy as a whole and, consequently, they frequently base their decisions on the influence exerted by neighboring relations. Such relations are, as discussed, likely to generate persistent aggregate volatility, because individual agents are systematically receiving the influence of different sentiment perspectives.

\section{Acknowledgment}

I would like to acknowledge the helpful comments offered by an anonymous referee. The usual disclaimer applies.

\section{References}

[1] G. Zschaler, Adaptive-network models of collective dynamics, Eur. Phys. J. Spec. Top. 211 (2012) 1-101.

[2] S. Boccaletti, V. Latora, Y. Moreno, M. Chavez, D.U. Hwang, Complex networks: structure and dynamics, Phys. Rep. 424 (2006) 175-308.

[3] D.J. Daley, D.G. Kendall, Stochastic rumours, J. Inst. Math. Appl. 1 (1965) 42-55.

[4] D.P. Maki, M. Thompson, Mathematical Models and Applications, with Emphasis on Social, Life, and Management Sciences, Prentice-Hall, Englewood Cliffs, NJ, USA, 1973.

[5] D.H. Zanette, Dynamics of rumor propagation on small-world networks, Phys. Rev. E 65 (2002) 041908.

[6] K. Thompson, R.C. Estrada, D. Daugherty, A. Cintron-Arias, A deterministic approach to the spread of rumors, Technical Report BU-1642-M, Cornell University, Dept. of Biological Statistics \& Computational Biology, 2003

[7] M. Nekovee, Y. Moreno, G. Bianconi, M. Marsili, Theory of rumor spreading in complex social networks, Physica A 374 (2007) 457-470.

[8] L. Huo, P. Huang, C.X. Guo, Analyzing the dynamics of a rumor transmission model with incubation, Discrete Dyn. Nat. Soc. (2012) 21. article ID 328151

[9] L.J. Zhao, J.J. Wang, Y.C. Chen, Q. Wang, J.J. Cheng, H.X. Cui, SIHR rumor spreading model in social networks, Physica A 391 (2012) $2444-2453$.

[10] Y.Q. Wang, X.Y. Yang, Y.L. Han, X.A. Wang, Rumor spreading model with trust mechanism in complex social networks, Commun. Theor. Phys. 59 (2013) 510-516.

[11] L. Zhao, J. Wang, R. Huang, H. Cui, X. Qiu, X. Wang, Sentiment contagion in complex networks, Physica A 394 (2014) 17-23.

[12] A.L. Barabási, R. Albert, Emergence of scaling in random networks, Science 286 (1999) 509-512.

[13] A.L. Barabási, Scale-free networks: a decade and beyond, Science 325 (2009) 412-413. 